\title{
Structuring multimedia data to facilitate decision making and reflection in product design
}

\author{
S. Phillips and J. T. McDonnell \\ University College London \\ Department of Computer Science, University College London, \\ Gower Street, London WC1E 6BT, UK \\ Telephone: +44 (0) 171267 1202, Fax: +44 (0) 1712842963 \\ Email:S.Phillips@cs.ucl.ac.uk, J.McDonnell@cs.ucl.ac.uk
}

\begin{abstract}
This paper describes the construction of a multimedia design resource, structured to reflect the design process. Initially an issue based representation was used to organise the multimedia data for a complete season's range of clothing in a sportswear company. This resource was used by the design team to review progress with the current season's design and to reflect on the process overall once it was completed. Based on experiences with making use of this resource, we constructed a system for capturing and re-using design knowledge which better suits product designers' priorities. We highlight the particular value of effectively structured multimedia material to design based companies where data is primarily visual in nature, where design cycles are rapid, and where brand consistency is important.
\end{abstract}

Keywords

Multimedia design resource, design rationale, decision support

\section{INTRODUCTION}

It is a widely held view that an ability to capture and retain knowledge used during product design is valuable to design based companies. The potential benefits of suitably structured multimedia resources could be enormous in design based industries where designers use old designs, samples and many diverse visual sources of material to inform and inspire their design activities (Phillips, 
1997). Much of the material referred to and created during product development forms an inherent part of the design rationale. This information may take many forms. As well as including data about previous products, competitors' products, sales information and marketing directions, there may be video sequences which encapsulate the essence of the product in some way and story-boards containing pictures and media samples intended to illustrate the product direction and design proposals.

One advantage of structuring and retaining information on design decisions and influences is that changes in membership of the design team might be accommodated with less disruption. This is particularly important when designing branded goods, as it can be extremely damaging to be inconsistent with brand image. In design orientated companies it is common for many prototypes to be created during the product development cycle, many of these are discarded and the investment in their development is also lost.

At present, the knowledge and information used during each product development cycle is not retained for future use. One reason making it difficult to preserve such data is that it is difficult to structure it in a way that will remain accessible over time. Much of the knowledge used in design is also tacit and therefore by its nature difficult to express. Furthermore, product designers are not traditionally required to be explicit about the rationale behind their designs and therefore a substantial amount of the information that they use during the design process is not shared with others or formally recorded.

In this paper we describe the construction of a multimedia design resource organised around the issues addressed during the design of a product range.

\section{DESIGN RATIONALE METHODS}

Moran and Carroll (1991) identified a number of reasons for capturing and representing design rationale, namely to enable designers to reflect on their reasoning at different stages in the design process, to facilitate communication of the design rationale to others, to enable the design knowledge to be reused or reflected upon in future design projects.

A number of notations have been developed for representing design rationale. These notations have been created mostly for the domains of engineering and software design. Although there are differences between the design processes in these domains and that of product design, there are enough similarities for these notations to provide a suitable starting point for product design rationale capture.

Much of the current research on capturing design rationale is based on variations of the more broadly applicable Issue Based Information System (IBIS), first described by Kunz and Rittel (1970). An IBIS is intended to 'guide the identification, structuring and settling of issues raised by problem solving groups.' (op. cit., p.1). Its application to the context of design deliberation, takes the form of a network representation of the various alternatives that are 
considered in response to issues and the arguments for and against the positions (Moran and Carroll, 1991).

MacLean et al (1996) developed a notation for representing design rationale called Questions, Options and Criteria (QOC). QOC is a notation for capturing what is considered during design which is also intended to encourage the exploration of design alternatives. A QOC representation is produced during the design process. It is based on the idea that a particular design is one which exists in a space of design possibilities and an analysis of the design space explains why the design emerged in the form it has from among the alternative possibilities. MacLean et al argue that creating a design space rationale 'will repay the investment in its creation by supporting both the original process of design and subsequent work on redesign and reuse by providing an explicit representation to aid reasoning about the design ..... serving as a vehicle for communication, for example among members of the design team' (op. cit., p.55).

It was not the intention in QOC to create a notation that would record the design deliberations per se, but rather to construct a representation of the design space that surrounds an object. The authors emphasise the fact that a QOC representation must be 'carefully crafted' itself and created along with the design specification. This makes it a potentially intrusive method on the designers' time. The merit of conducting a design space analysis before commencing a design is that it provides a designer with a detailed analysis of the design alternatives. However certainly in product design, the time factor would often be prohibitive to such analysis. The IBIS notation not only requires less effort to construct but also does not require the designer to deviate far from the usual design tasks and provides a more natural representation of the design rationale.

Lee and Lai (1996) have devised a notation for representing decision rationale called DRL (Decision Representation Language). However they highlight the fact that there are limitations in the scope of DRL in so far as it is primarily a model for capturing decision making and it does not capture certain aspects of design rationale. This consideration and the complexity of the representation was also an inhibiting factor for us as we wanted to give the designers something relatively simple to work with initially.

QOC shares the same drawback of complexity with DRL, although less so. An IBIS like approach presents a fairly natural way of structuring a design rationale since it allows issues and their arguments to be presented as they are discussed and does not artificially force the designers to be explicit about criteria to which they would not otherwise have paid any attention.

In summary, three of the prominent existing methods for representing design rationale were evaluated: DRL, QOC and IBIS. We concluded that IBIS provided the most appropriate starting point due to the simplicity of its representation and the ability of the model to represent the natural process of product design. A further important advantage of the IBIS model is that it 'is geared to capturing deliberation as it happens,'(Moran and Carroll, 1991, p.198) and it does not require the rationale to be constructed as a separate part of the development process. 


\section{CONSTRUCTING THE INITIAL KNOWLEDGE BASE}

We conducted a detailed study to capture the design rationale for a Spring/Summer 1998 product range for an American Sportswear company. Subsequently an issue based structure was constructed using the data collected. The aim of this knowledge base was to store all the information relating to the products being designed and to capture the decisions relating to their development. An IBIS-like representation was used as an initial basis from which to investigate the real problems of design rationale in the field of product design.

The notation consisted of nodes and links to show relationships between them. The nodes represented issues, positions (ideas) about how to address the issues and arguments for and against the positions. These could be simply linked to one another in a logical manner. In addition, the notation allowed issues to be directly linked to other issues and positions to be linked to other positions. (This latter extension to IBIS was originally proposed by Conklin and Begeman (1988)). A decision node type was also incorporated into the notation which could be linked to issues or positions, this too is an extension of the original IBIS notation. In the remainder of this paper we refer to this notation as IBIS $^{+}$for simplicity.

To explore the requirements for useful representation (maps) of the collected design knowledge we used a software environment that supported a graphical representation of the design rationale and which allowed us to link multimedia data to the nodes of the maps. Each node could contain some explanatory text and could be connected to other reference files launched directly from the nodes, such as other IBIS ${ }^{+}$maps, graphic, video or audio files. The resulting structured collection of data was installed on the computers belonging to the design team incrementally as it was being constructed. An analysis was then conducted to assess its effectiveness and limitations.

Showing the designers an insubstantial prototypical representation, comprising a small quantity of data to which they find it difficult to relate and asking them both to imagine a realistic, substantial set of useful data and to conjecture how they might make use of it, is asking too much. In order to gain quality reflection and responses from the designers the maps needed above all to be realistically complex, capturing as much knowledge as possible, thereby making them relevant in the real design setting. It was therefore essential that the representation should be comprehensive and include a full season of design data in order to make our findings credible. To achieve the necessary complexity and detail we followed a complete product development season. All of the formal design meetings were attended and fully transcribed, and further visits were made to members of the product development team to collect other background material that had not been gleaned in the formal design meetings. In addition detailed interviews were carried out to obtain feedback on the value of the IBIS ${ }^{+}$ knowledge structure and to assess its effectiveness. 
The IBIS ${ }^{+}$knowledge structure contained approximately 200 image files, 4 video files, 2 audio files, and 30000 words of design meeting transcripts. It consisted of 13 IBIS $^{+}$maps containing 480 nodes and 471 links.

\section{ANALYSIS OF THE INITIAL KNOWLEDGE STRUCTURE}

The designers used the design rationale as it was being constructed during the design season, to reflect on what they had stated they would achieve at the initial meetings and to identify what decisions had for various reasons fallen by the wayside. At the end of the design season when the IBIS ${ }^{+}$maps were completed the product design team experimented with them for a few weeks. Then a detailed analysis of their reflections and opinions was conducted.

Concern was voiced regarding the ability to capture all the design decisions, despite the fact that as far as possible all the formal design meetings had been attended. Although things not explicitly expressed cannot be captured, one might expect that decisions of import would be shared or discussed in the formal design meetings. Unfortunately however, this is not always the case. For example design issues may be discussed between a sub group of the design team on the way to meetings or in other non scheduled discussions. Furthermore, urgency sometimes dictates that decisions have to be made outside of formal meetings. This may sometimes mean that there is no time to consult and inform the rest of the design team about some of the decisions made. This is an inevitable limitation of any attempt to capture real decision making. On a related issue Fischer has pointed out that, 'a truly complete account of the reasoning relevant to design decisions is neither possible nor desirable. It is not possible because some design decisions and the associated reasoning are made implicitly by construction and are not available to conscious thinking. Some of the rationale must be reconstructed after design decisions have been made. Many design issues are trivial; their resolution is obvious to the competent designer, or the design issue is not very relevant to the overall quality of the designed artifact. Accounting for all reasoning is not desirable because it would divert too many resources from designing itself.' (Fischer et al, 1996, p.270)

To address these inherent limitations we propose a reflective stage at the end of the design process where the decision structure is reviewed, corrected, annotated and updated in order for the knowledge representation to be of value for future reference. In our study we found that this task does not need to take more than a couple of hours and ensures the design rationale structure is up to date. It is also a useful exercise to refresh the memories of the design team about the process that has been gone through and the goals that have or have not been achieved.

One of the major comments was that it would be extremely useful for the current design rationale to be used as a template to start building the next season's range. However the maps editor was not at an advanced enough stage for the designers to be able to develop a template for themselves. There were many other important findings from the analysis of the designers' experience with using 
our IBIS ${ }^{+}$maps. These concerned weaknesses in the $\mathrm{IBIS}^{+}$notation; how to support reflection during and after the design process; interface requirements ways of presenting data from the maps for specific purposes: and direct benefits to the design team and the company of retaining knowledge used during the design process over a succession of design cycles.

The limitations of the IBIS ${ }^{+}$notation led to a complete restructuring of the material collected for the whole design season. The adaptation of the notation and its extension to cope with the realities of the product design process are described in a separate paper (Phillips and McDonnell, 1998). A section of one of the maps which uses the improved notation, which we refer to as DR maps, is shown in Figure 1. In the remainder of this paper we discuss those issues concerning product design which most affect the way the multimedia design data is structured to be most useful.

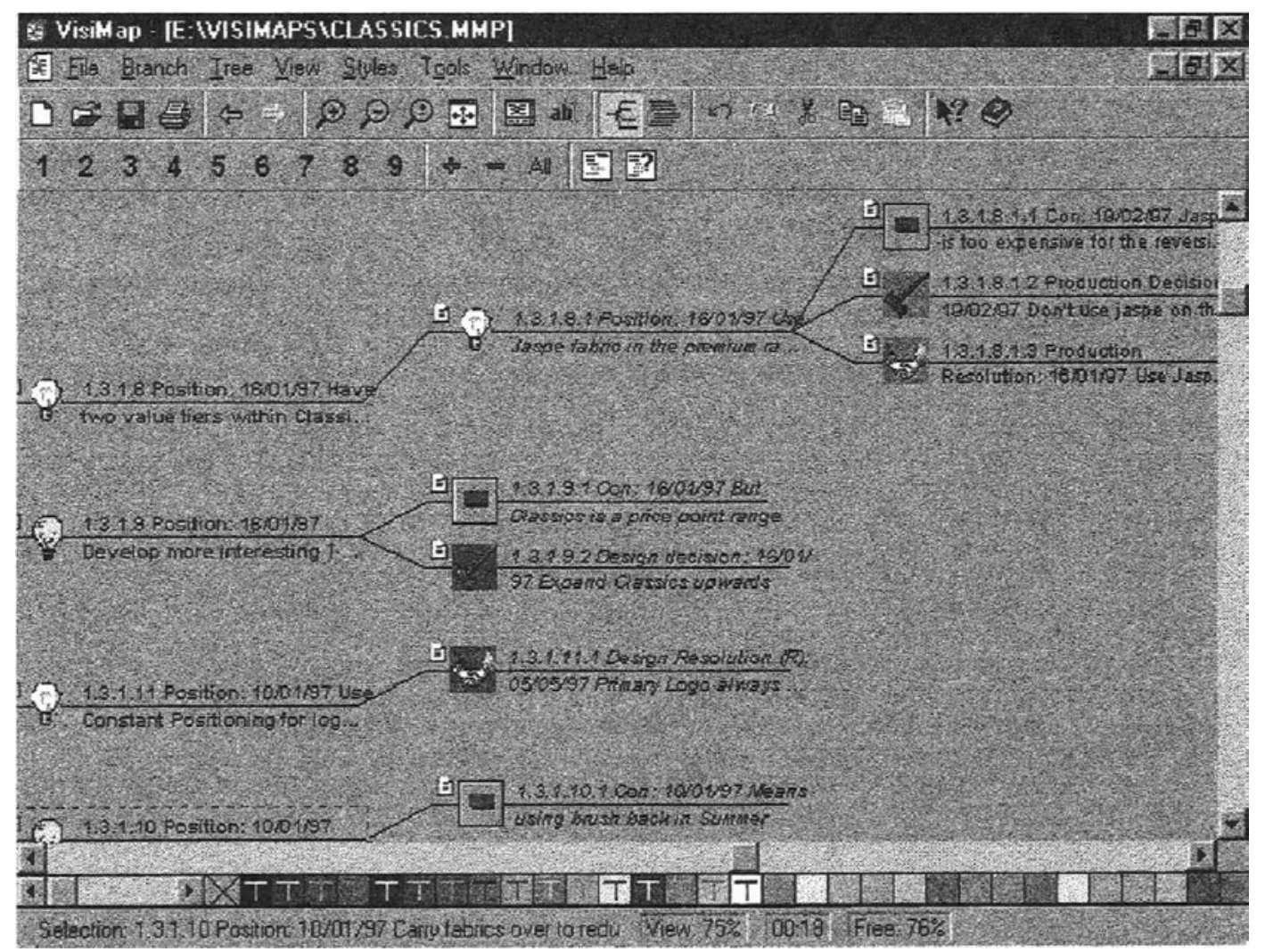

Figure 1 A section of a design rationale map.

\section{STRUCTURING MULTIMEDIA DATA INTO A TOOL TO SUPPORT DESIGN DECISIONS}

Several issues were raised concerning the capability of the tool to support reflection on the design process, which in turn would have an impact on the design decision making in the next season's product development meetings. It 
quickly became apparent that there was a requirement for the knowledge structure to be used not only as a record of the design rationale but also to enhance strategic planning and to provide a starting point for the following season's design cycle.

A particularly useful aspect was thought to be the use of the knowledge structure as a reflective tool to enable the design team to review the previous range development process and to highlight where goals had been achieved. The DR maps sparked debate within the design team as to why certain issues had remained unresolved or had fallen by the wayside. The design team were given access to the knowledge structure as it was being built, and while the design process was still ongoing. They noted that it was useful to use the maps to see what goals had been achieved and identify what had been accomplished and what had not, even before the design process had been completed. Figure 2 shows how visuals of products that are discarded from the range at an early stage are retained within the multimedia maps in order that the designers may refer back to them at a later date. The knowledge structure provided an insight into the reasoning behind certain decisions being made and allowed the identification of the issues that were lost during the design process. The design team also noted that it would be a useful historical record, as over time catalogues and samples get mislaid. Many claims are made about the importance of recording the design process, but in the field of product design little research has been conducted to substantiate this.

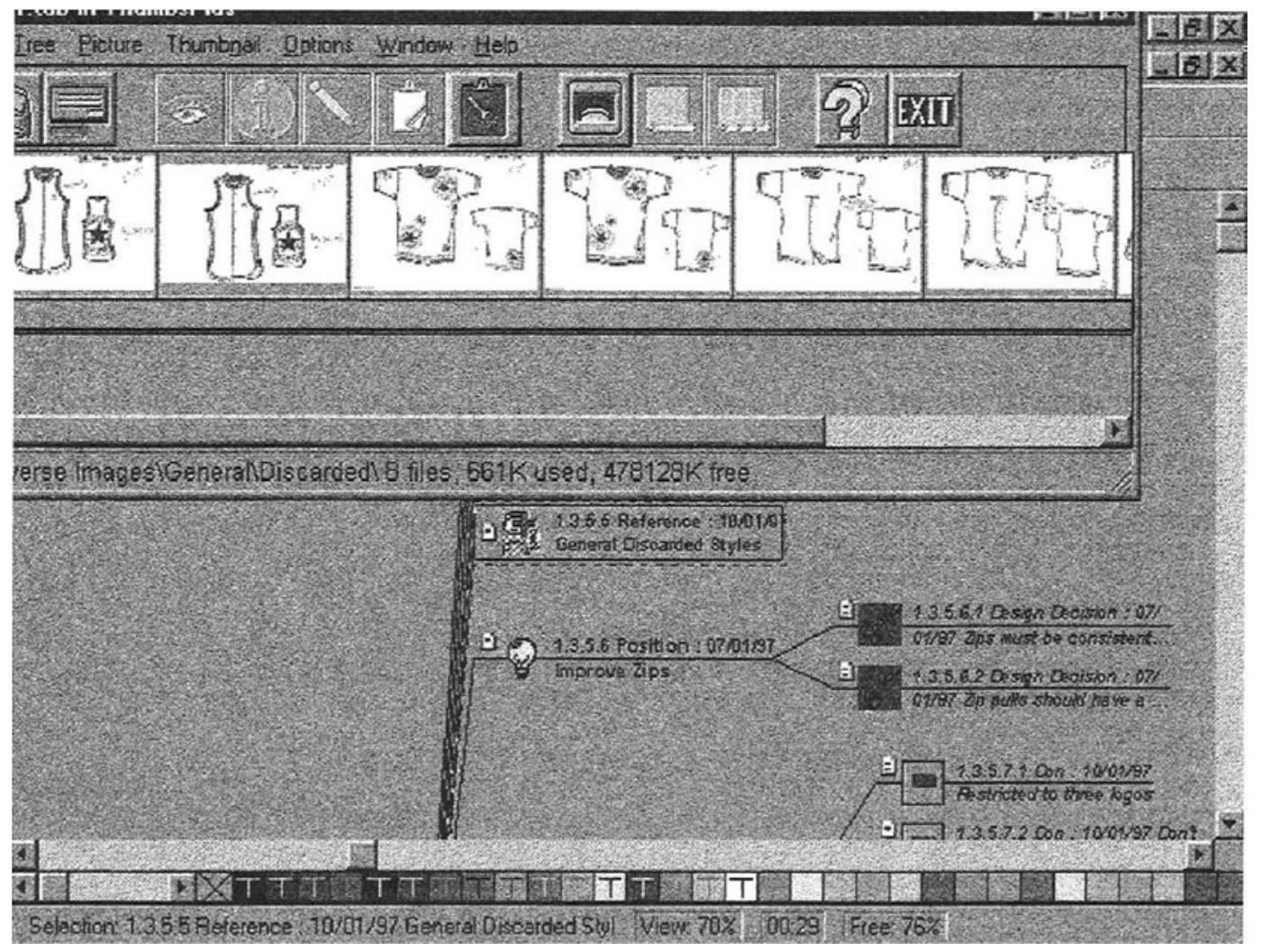

Figure 2 Visuals of discarded designs contained within the maps. 
It was further commented that the knowledge structure provided a useful starting point for the next season's range planning. It enabled a review to be quickly conducted of the goals identified at the beginning of the previous season and a new set of goals to be drawn up for the coming season. It became apparent that the decision structure would not change entirely from season to season. Some of the design decision structure would be unique each season, but a relationship would exist with the structure from the previous season. The design team wanted to use the current season's DR maps as a template for the next season's maps to provide a starting point and to reduce the work of constructing the next season's design rationale. A set of DR maps would be used initially to identify a model path for the product development. From this a template could be created to form a basis for the following season's maps. This would make the knowledge base a very useful tool for design/product managers and it would speed up and enhance the following season's range planning. However, a reflective stage would be required first, in order to 'tidy up' and annotate the maps with decisions not made explicit in the design meetings. Given the correct tools this is a modest task, although it would need to be done as soon as possible at the end of the design process. The design team felt it would provide invaluable support for their strategic planning and decision making at the start of the following seasons design cycle. Figure 3 demonstrates how a template will be created after a reflective stage at the completion of a development season.

Another issue that was raised was that every time a design cycle has been completed, a link to the next season's map should be added so that the design rationale can be traced through seasons. One reason that not all the rationale was captured in the DR maps was that many of the design scenarios understood by the design team, stemmed from previous season's development. Over a period of time these omissions could be overcome to some extent by linking in seasonal progression. Exploration of the decision structure through a number of seasons would give an understanding of previous design scenarios and decisions.

Specific interface facilities were identified which would enhance the usefulness of the DR maps for making decisions about future seasons' product development. Some of these were:

- Ability to select all products from the current range that are being carried over into the following season's range (to import them directly into the new DR maps).

- Provision of a gallery summary of a previous range alongside a working screen for the current range.

- Slide show display of the path of evolution of any product selected from a gallery display. 


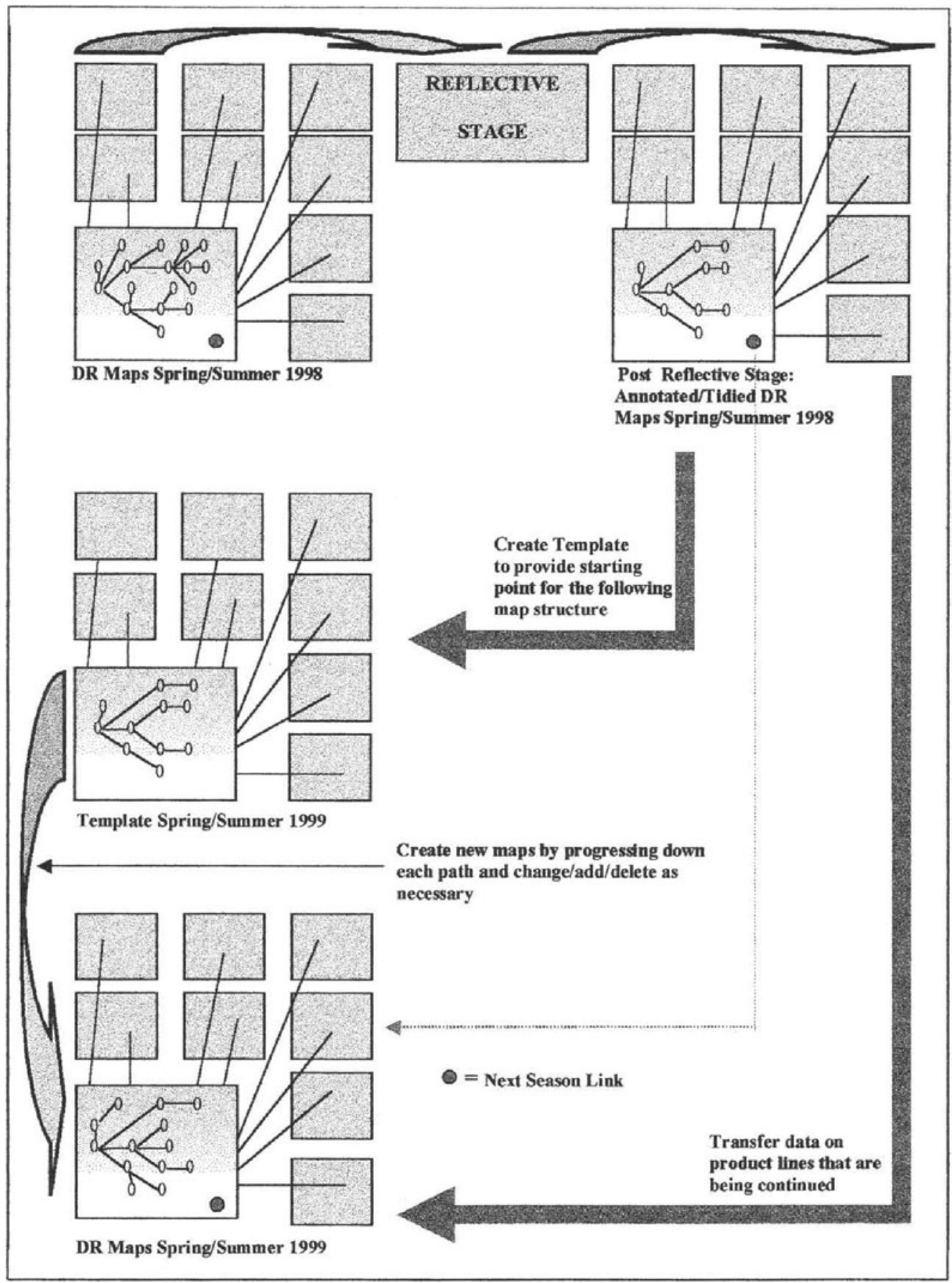

Figure 3 Reflective stage adjustments followed by template creation. 
As a source of reference data allowing historical exploration and as a decision making tool for the design team in years to come, the maps are currently presented with the final products as the end result in the decision trees that the maps represent. For use as a historical resource it is more sensible to be initially presented with the final range of products, and then to be able to go through the decision making process in reverse to see the evolution from the original inspiration. Graphics from each range should be viewed together or in categories of the various stage of product development, e.g. photos, coloured sketches, and line drawings. The designers prefer a slide show style display of the lines of development starting from the ends of the branches on the maps, so that any particular product can be taken individually and all the images along its development path can be viewed starting with the final photo of the finished product, as demonstrated in Figure 4.

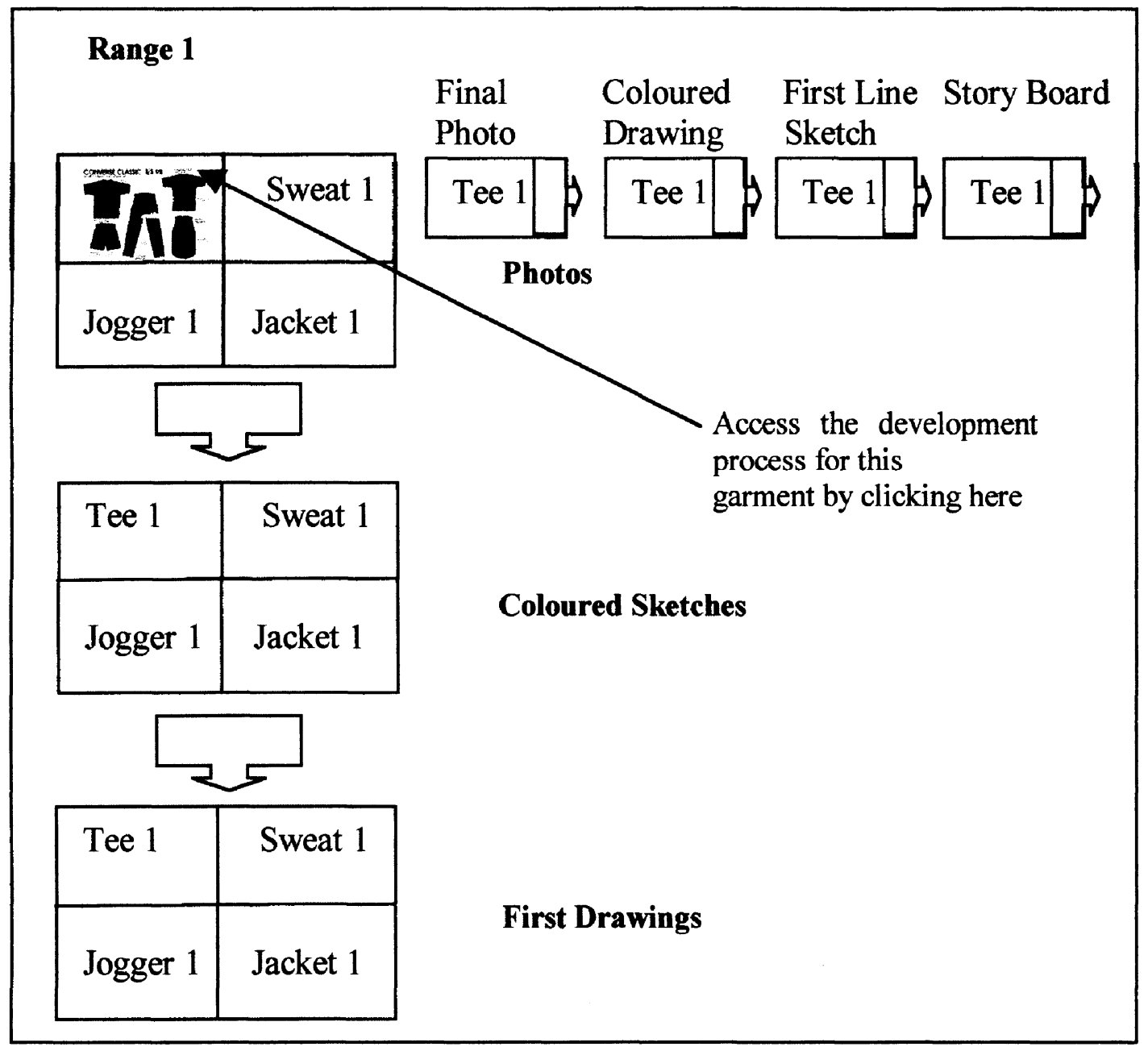

Figure 4 Displaying the lines of development for each product.

In product design much implicit knowledge is visual data of a multimedia nature, which we believe can be usefully retained for later reference by setting it in context in a DR map structure. Figures 5 and 6 demonstrate the potential for capturing tacit knowledge by including visual data within the DR maps. 


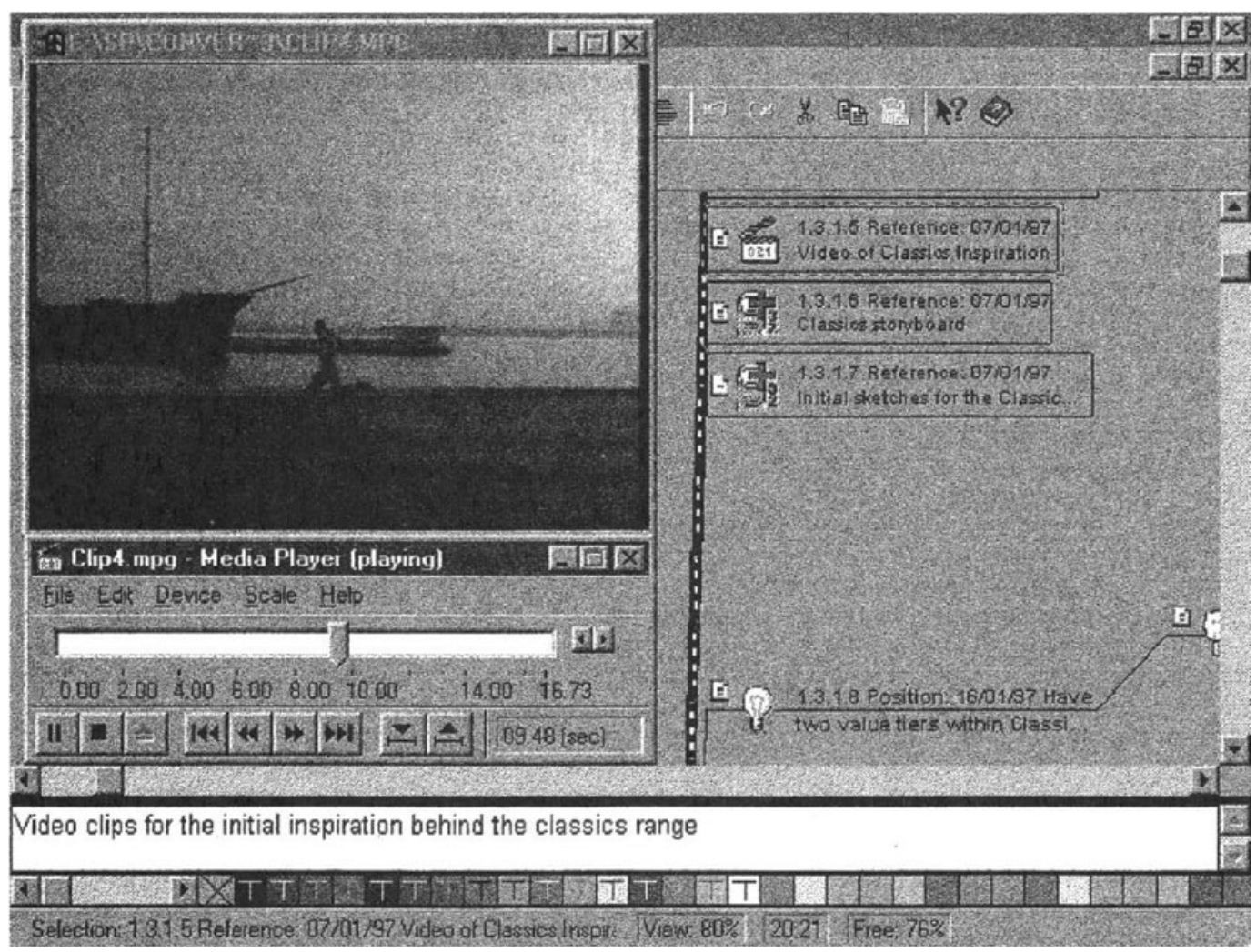

Figure 5 A clip from one of the inspiration videos contained with the maps.

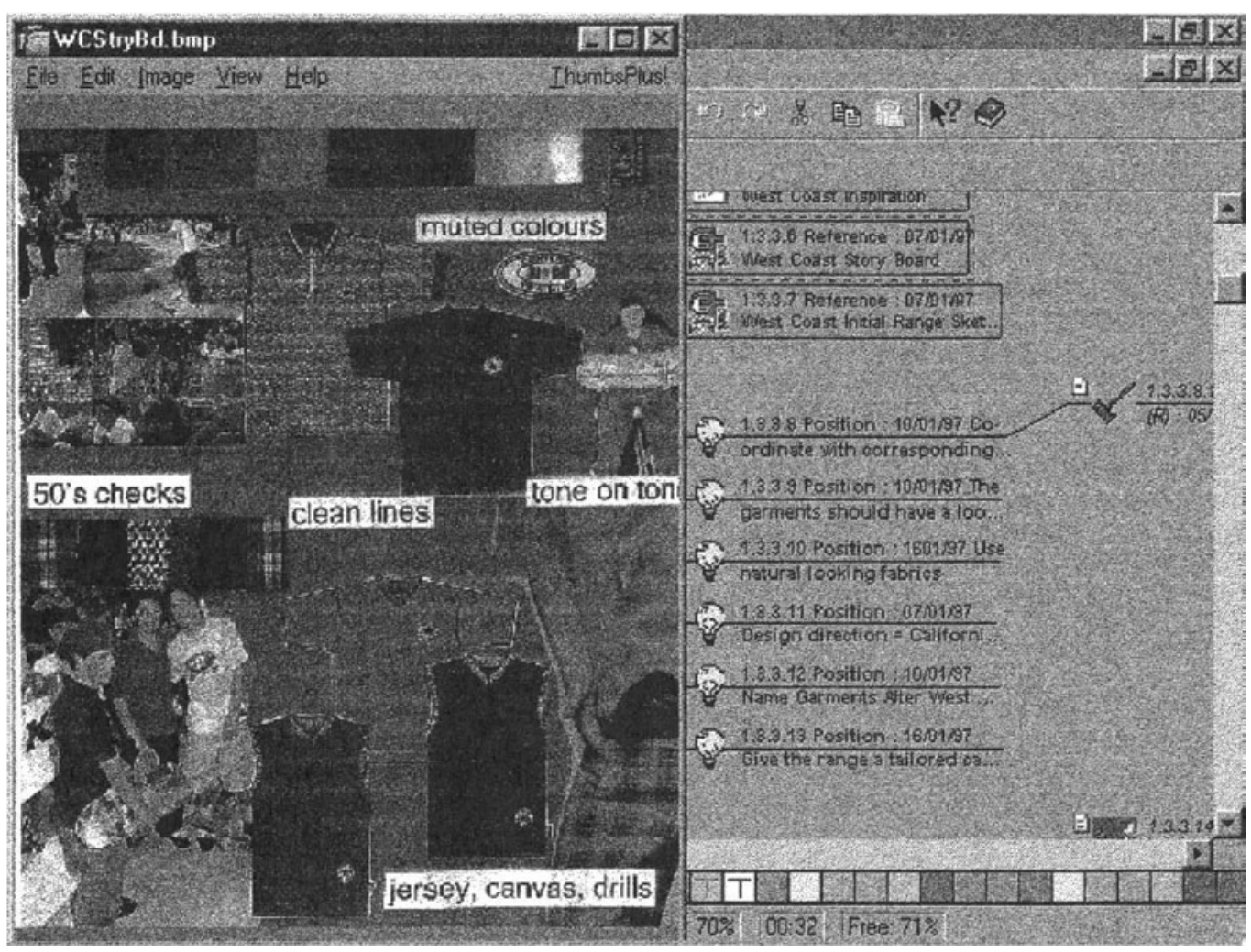

Figure 6 A visual design story board within one of the maps. 


\section{CONCLUSION}

The initial reaction of the design team to the DR maps was how well they graphically demonstrated the work conducted and the processes and decisions undertaken during the product development cycle. Having experimented with the resource, it became clear to the product development team that once the design rationale had been captured over a few seasons the benefits would become more significant.

Our research identified many positive benefits from constructing as full a representation as possible of the design rationale. These included:

- Aiding and speeding up the planning for the following seasons ranges.

- Ensuring higher quality decisions are made on the new ranges on the basis of reflection on the previous design season.

- Providing new team members with a history of previous product development and design decisions.

- Helping branded products to retain consistency in their brand identity.

- Allowing team members to analyse their own personal development.

- Providing an archive of previous design that will become increasingly useful with the passing of time as a resource for visual research/inspiration.

- Providing a resource for marketing activities that may incorporate 'retro' designs. Many branded clothing companies such as Levis use old, archived design work from previous decades in their 'lets return to our roots' marketing activities.

- Aiding quality reflection on both aesthetic and technical decisions. It is useful to explicitly represent design rationale in those product design domains where the over-riding decisions may be split into those which are aesthetic in nature and those addressing production/technical concerns. For example, a certain style may be adopted due to the design team's belief in it having the correct aesthetic values for the market and yet later prove to be a 'dog' in terms of sales. In such a situation if a design rationale representation had been constructed it might lead the design team to question their judgement on whether they were using the correct aesthetic values for the market place with this product. Similarly it would also be useful to have a rationale of technical/production decisions. In a case where a fabric proves to be unsatisfactory, resulting in a high number of product returns, the specification and rationale for choosing that fabric could be referred to when seeking a sensible resolution.

Ideally the design rationale would not be built by one person and then presented at the beginning of each meeting as it was in our work. We envisage it being constructed as an integral part of the design meetings by all members of the design team. Members of the team should be allowed to view or add to the rationale at any time and it should be used as a reference point during meetings 
for the team to reappraise goals and objectives. The nature of product design means that much of the tacit knowledge understood by the designers would be contained in the predominantly visual multimedia data linked to the design rationale structure. Although this data may not mean anything to the untrained eye, to an experienced designer it conveys a lot of valuable information and adds an expressive new dimension to formal design rationale notations. In clothing product design in particular, a range of similar products are created season after season, and there are two or three seasons each year. The amount of design reuse prevalent means that it appears to be much more practical to justify the effort of capturing design rationale in this sort of domain than in to the more traditional domains of software or engineering design.

More research is required to assess the feasibility of design orientated companies in general allocating adequate resources for capturing and retaining product development rationale over a substantial period of time. Nevertheless, it is clear from our research that if a company invests the time and money into collating and structuring design rationale they may be rewarded with a multimedia resource that will enhance their design decision making process. It would provide a more efficient approach to their product design and when collated over a number of design seasons could be used further to support their overall strategic planning.

\section{REFERENCES}

Conklin, J. and Begeman, M. L. (1988) gIBIS: A Hypertext Tool for Exploratory Policy Discussion. ACM Transactions on Office Information Systems, Vol. 6, No. 4, October 1988, pp.303-331.

Fischer, G., Lemke, A.C., McCall, R., and Morch, A.I. (1996) Making Argumentation Serve Design. pp.267-291 in Moran, T.P. and Carroll, J.M. (1996) op. cit.

Kunz, W. and Rittel, H.W.J. (1970) Issues as Elements of Information Systems. Working paper No. 131, Institute of Urban and Regional Development, Univ. of California, Berkeley, California, USA.

Lee, J. and Lai, K. (1996) What's in Design Rationale? pp.21-51 in Moran, T.P. and Carroll, J.M. (1996) op. cit.

MacLean, A., Young, R.M., Bellotti, V.M.E. and Moran, T.P. (1996) Questions, Options and Criteria: Elements of Design Space Analysis. pp.53-105 in Moran, T.P. and Carroll, J.M. (1996) op. cit. 
Moran, T.P. and Carroll, J.M. (1991) Special Issue on Design Rationale. Human Computer Interaction, Volume 6, Numbers 3 and 4, 1991, LEA, New Jersey, USA.

Moran, T.P. and Carroll, J.M. (eds.), (1996) Design Rationale - Concepts, Techniques and Use, LEA, New Jersey, USA.

Phillips, S. (1997) Multimedia Fabric Libraries for the Textile and Clothing Industry. Proceedings of $\mathbf{7 8}^{\text {th }}$ World Conference of The Textile Institute, Volume 1, Thessaloniki, Greece, May 1997. The Textile Institute, Manchester, UK pp.397-431.

Phillips, S. and McDonnell, J.T. (1998) Empowering The Design Team: A Multimedia Design Resource To Facilitate The Capture, Retention, And Reuse Of Knowledge Acquired During Product Development. Forthcoming paper at the Design Research Society conference, Quantum Leap, Birmingham, $8^{\text {th }}-10^{\text {th }}$ September 1988.

\section{BIOGRAPHY}

\subsection{Sophie Phillips}

Sophie Phillips is working on a $\mathrm{PhD}$ in the Department of Computer Science at University College London. She has a BA in product development from the London College of Fashion and has worked in the buying sector of the clothing industry. Her research interests focus on the development of information systems to provide design support during the product design and development process.

\subsection{Dr. Janet McDonnell}

Janet McDonnell is a senior lecturer in the Department of Computer Science at University College London. Her research interests include design support systems, knowledge management and strategic uses of I.T. for information management. 